Revue d'histoire de l'Amérique française

REVUE D.HISTOIRE DE L'AMÉRIQUE FRANÇAISE

\title{
François Blanchet et le mouvement réformiste en médecine au début du XIX ${ }^{\mathrm{e}}$ siècle
}

\section{Jacques Bernier}

Volume 34, numéro 2, septembre 1980

URI : https://id.erudit.org/iderudit/303857ar

DOI : https://doi.org/10.7202/303857ar

Aller au sommaire du numéro

Éditeur(s)

Institut d'histoire de l'Amérique française

ISSN

0035-2357 (imprimé)

1492-1383 (numérique)

Découvrir la revue

Citer cet article

Bernier, J. (1980). François Blanchet et le mouvement réformiste en médecine au début du XIX ${ }^{\mathrm{e}}$ siècle. Revue d'histoire de l'Amérique française, 34(2), 223-244. https://doi.org/10.7202/303857ar d'utilisation que vous pouvez consulter en ligne.

https://apropos.erudit.org/fr/usagers/politique-dutilisation/ 


\title{
FRANÇOIS BLANCHET ET LE MOUVEMENT RÉFORMISTE EN MÉDECINE AU DÉBUT DU XIXe SIÈCLE
}

\author{
JACQUES BERNIER \\ Département d'histoire \\ Université Laval
}

\begin{abstract}
A professional group is a product of the society in which it appears, as well as of its own tradition of learning. Such a guide influences and is influenced by that society as a whole and its history is layely the story of these relationship. ${ }^{1}$
\end{abstract}

\section{Introduction:}

Le Bas-Canada a connu, au début du XIXe siècle, une réponse importante dans le domaine de la médecine. Cette période marque en effet, le moment d'émergence de certains traits qui vont rester caractéristiques de ce secteur d'activité jusqu'à nos jours.

Un premier aspect de cette réforme est lié à la mise en place du processus de professionnalisation. Les praticiens civils cherchent alors à développer leur influence comme groupe. Ils commencent à s'organiser en association et à revendiquer une plus grande autonomie à l'égard du pouvoir politique et des praticiens militaires de la colonie. On voit aussi l'élite médicale demander le pouvoir, pour le corps médical, de délimiter les frontières de son domaine d'activité et d'exercer un plus grand contrôle sur les personnes appelées à pratiquer la médecine. En conséquence, le corps médical commence à demander le droit de définir lui-même les normes d'accès à la profession et le pouvoir d'évaluer la compétence des candidats.

1 R.H. Shryock, Medicine and Society in America, 1660-1860 (3e éd., Ithaca, Cornell Univ. Press, 1975), 2. 
Les médecins civils sont aussi à la recherche d'une reconnaissance sociale qu'ils chercheront à se donner par le biais, entre autres, de l'établissement de curricula de formation uniformes et la création de cours médicaux qui permettront non seulement de hausser et d'uniformiser le niveau des connaissances du groupe, mais d'instituer ainsi une production standardisée de praticiens de la santé $^{2}$. De même, des efforts sont faits pour assurer une meilleure formation pratique et l'observation directe sur les patients. Ces efforts aboutiront, un peu plus tard, à la mise en place de l'enseignement clinique dans les hôpitaux.

Enfin, troisième aspect de cette réforme, on voit le corps médical s'impliquer de plus en plus dans le domaine de la santé publique (prévention et organisation des soins des malades). Cette action s'explique bien sûr, en grande partie, par les possibilités accrues (nombre plus élevé de médecins diplômés et techniques nouvelles) qu'offre la profession médicale pour faire face à une recrudescence des maladies et des problèmes sociaux à cette époq̣ıe, mais auıssi parce que ce sera l'occasion pour ce groupe de confirmer et d'accroître ses possibilités d'intervention dans un secteur jusque-là négligé et qui lui échappait en grande partie. Cette réforme, il convient donc de la concevoir comme un effort de démocratisation et de modernisation des institutions médicales de la colonie, joint à une stratégie de promotion collective et individuelle.

Les objectifs de ce texte sont donc de rendre compte de cette réforme, de montrer le contexte dans lequel elle s'inscrit et de mettre en évidence le cheminement et les stratégies de quelques acteurs de ce projet, notamment François Blanchet qui fut un de ses artisans à Québec ${ }^{3}$.

2 Larson écrit à ce sujet: «cognitive standardization is a most powerful generator of deeply shared cultural assumptions». The Rise of Professionalism (Berkely, Univ. of California Press, 1977), 46.

3 Cette recherche a bénéficié d'une subvention du Ministère des Affaires culturelles du Québec. L'auteur tient à remercier MM. Yves Roby et Jean-Pierre Wallot de leurs commentaires. 


\section{1 - LA FORMATION ET LES DÉBUTS PROFESSIONNELS}

\section{a) Les études}

François Blanchet est né en $1776^{4}$, d'une famille d'agriculteurs aisés de Saint-Pierre-de-la-Rivière-du-Sud ${ }^{5}$, sur la rive sud du SaintLaurent, non loin de Québec. Brisant avec une longue tradition d'agriculteurs il montre, dès son adolescence, une préférence marquée pour les études, voie que suivront, du reste, plusieurs Blanchet des générations suivantes.

Après avoir reçu une formation élémentaire dans son village natal, François alors âgé de 15 ans, s'inscrit en 1790 au Séminaire de Québec, pour y faire des études classiques ${ }^{6}$. Elles durent quatre ans et il les fait sans grandes difficultés ni succès éclatants. Le temps venu de faire le choix d'une carrière, il opte pour la profession médicale et demande à être reçu, pour faire son apprentissage, chez l'un des médecins les plus en vue de l'époque: James Fisher. Ce dernier est en effet un des grands noms du corps médical du district de Québec qui compte à l'époque une vingtaine de praticiens reconnus par les autorités ${ }^{7}$.

Ce James Fisher, en plus d'être chirurgien de la garnison de Québec, est médecin des religieuses de l'Hôpital Général et il bénéficie d'une vaste clientèle dans la ville ${ }^{8}$. Sa renommée dépasse même le plan local.

En 1874, alors qu'un comité est formé par le Conseil législatif afin d'étudier différents problèmes relatifs au peuplement de la colonie et au développement de l'agriculture et de la colonisation ${ }^{9}$, C. Blake de Montréal et J. Fisher, qui ont été consultés, saisissent l'occasion qui leur est offerte pour faire part au Gouverneur des problèmes qui sévissent dans le secteur médical. Fisher, quant à lui, rédige un mémoire dans lequel il avance l'idée que si la province

${ }^{4}$ Extrait du registre des baptêmes de la paroisse de Saint-Pierre-du-Sud, 14 avril 1776, comté de Montmagny.

5 Son père est à l'aise et certains de ses frères remportent des prix d'agriculture. $L a$ Gazette de Québec, 21 décembre 1818, 7 septembre 1820,28 août 1823 . Pourtant son père et au moins un de ses frères ne savent pas signer. A.N.Q.Q. notaire Lelièvre, 26 septembre 1817 , no 9981 , et 8 octobre 1798 , no 1004 .

6 A.S.Q. «François Blanchet».

7 Almanach de Québec pour l'année 1791 (Québec, J. Neilson, 1791), 73.

$8 \mathrm{M}$. Abbott, History of Medicine in the Province of Quebec (Montréal, McGill University, 1931), 46

9 A Shortt et A.G. Doughty, Documents concernant l'histoire constitutionnelle du Canada 1759-1791 (Ottawa, Impr. du Roi, 1911), 610-611. 
comptait déjà davantage de médecins compétents, cela pourrait contribuer considérablement à faire baisser la mortalité. Se plaignant avec insistance du problème des charlatans dans les campagnes, il rappelle qu'aucune législation n'est encore venue ${ }^{10}$ régler la pratique de la médecine et que «quiconque, même des gens sans connaissance, sans étude et sans moralité» peut, dans cette province, ouvrir un cabinet médical ${ }^{11}$. A son avis, des mesures urgentes s'imposent: il demande qu'on exerce un contrôle plus strict des personnes habilitées à pratiquer la médecine dans la province (médecins, chirurgiens, sages-femmes, apothicaires) et que des bureaux d'examinateurs soient institués à cet effet. C'est du reste à la suite de cette proposition que sera passée l'ordonnance de 1788 qui constitue la première loi régissant la pratique de la médecine sous le Régime anglais. Par cette loi, le gouverneur autorise la pratique de la médecine aux seuls candidats qui subissent avec succès l'examen passé devant les bureaux d'examinateurs de Québec ou Montréal créés la même année ${ }^{12}$.

C'est donc chez un médecin en vue que Blanchet s'engage en 1795 pour y faire son apprentissage. Il y reste quatre ans et c'est là qu'il reçoit la première partie de sa formation médicale, qu'il apprend l'anglais et qu'il est mis en contact avec les personnalités les plus influentes du monde médical et même politique de l'époque.

Comme bien d'autres jeunes de la fin du XVIIIe siècle, Blanchet aurait pu arrêter là ses études et se présenter à l'examen du bureau des examinateurs de son district. Mais Blanchet voit plus grand. Du reste la bonne situation financière dont il jouit, grâce à l'héritage de près de 8000 livres $^{13}$ que son père lui a laissé quelques années auparavant, lui permet de pouvoir continuer ses études. Disposant de tous les atouts nécessaires: une bonne formation secondaire et un apprentissage chez un maître reconnu, disposant de suffisamment d'argent et parlant l'anglais, François décide donc, en 1799, d'aller poursuivre ses études à l'étranger, au Collège Columbia de New York où il restera jusqu'en 1801.

\footnotetext{
10 Depuis 1760 .

11 Ce texte de Fisher a été publié dans M.J. et G. Ahern, Notes pour servir à l'histoire de la médecine dans le Bas-Canada (Québec, s.e. 1923), 217 à 223.

12 Les candidats qui ont fait leurs études dans des universités britanniques ou qui ont servi pendant plus de trois ans dans l'armée ou la marine sont aussi reçus sans examen.

13 Constitué en terres qu'il vend en 1798 à son frère. A.N.Q.Q., notaire Lelièvre, 8 octobre 1798, no 1004. Vente de terrains de François Blanchet à Joseph Blanchet.
} 
Ces années sont sans aucun doute les plus décisives de sa vie. C'est là qu'il complète sa formation médicale, qu'il se rode aux idées démocratiques américaines ${ }^{14}$ et qu'il obtient son diplôme universitaire. Ce diplôme, d'ailleurs, il l'a bien mérité car ces deux années passées à New York, il les a entièrement consacrées à l'étude et à la recherche. François Blanchet est un étudiant sérieux, studieux et ambitieux. Ses lettres à John Neilson, son mentor, révèlent le souci qu'il a de s'affirmer sur le plan scientifique et de réussir sur le plan professionnel. A New York donc, en plus de suivre des cours, il prépare une thèse. Cette thèse qu'il intitule «Recherches sur la médecine ou l'application de la chimie à la médecine» s'inscrit dans le débat sur l'économie animale, thème qui fut à la mode plus tôt en Europe et qui s'étend maintenant aux États-Unis. Il écrit cette thèse en moins de deux ans et trouve à New York un éditeur qui la publiera en français en $1800^{15}$.

La médecine n'est pas cependant la seule discipline qui retient son attention; il a également un penchant pour les sciences naturelles et plus particulièrement pour la minéralogie et la physique. Bien qu'il ne publiera rien sur ce premier sujet, il lit et achète des livres ${ }^{16}$. Les problèmes relatifs à l'origine de la lumière et des aurores boréales sont, en particulier, des sujets que Blanchet affectionne, et sur lesquels il a des opinions précises. Il présente, du reste, sur ces sujets, deux mémoires à l'American Philosophical Society de Philadelphie dont il fait partie. Blanchet, dans ses écrits, s'est choisi un adversaire de taille; il veut démontrer que la théorie de Newton sur la lumière est fausse. Blanchet est convaincu que la lumière n'est pas une émanation du soleil mais bien un élément qui, comme l'eau, passe alternativement à l'état de solide, de fluide et de gaz. En corollaire à cette thèse, il n'hésite pas à soutenir non plus que les aurores boréales ne sont pas produites, comme les Newtoniens le prétendent, par des fluides électriques issus du soleil, mais résultent de la condensation de la lumière par le froid ${ }^{17}$. Outre sa participation à cette société savante, François Blanchet collabore aussi à une

14 «He brought principles of purest democracy». H.T. Manning, The Revolt of French Canada, 1800-1835 (Toronto, 1962), 56.

$15 \mathrm{Fr}$. Blanchet, Recherches sur la médecine ou l'application de la chimie à la médecine (New York, Parisot, 1800).

16 Lettre à J. Neilson, N.Y., 22 mars 1801. Coll. John Neilson, A.P.C., M.G. 24-31, 265 sq. En décembre 1802, il achète les 5 volumes du chimiste Fourcroy, collaborateur de Berthollet et Lavoisier, ibid., M.G. 24-B1, 1: 395.

17 Ibid. 
revue médicale, The New York Medical Repository, pour laquelle il écrit quatre brefs articles ${ }^{18}$.

Avec François Blanchet, nous sommes donc en présence d'un homme qui a voulu se donner une bonne formation, qui suit de près le développement de la vie scientifique de son époque, et qui cherche à établir des contacts et à communiquer avec les membres des milieux savants. Bien qu'il considère que «théoriser» constitue un défaut majeur des philosophes et des savants de son siècle, lui aussi cherche à se faire valoir, au sein de ces milieux, par une théorie. En s'excusant presque, il rapporte à Neilson, en 1880, que lui aussi a la «démangeaison de systématiser» et qu'il n'a pu s'empêcher de «rédiger son petit système sur la médecine» ${ }^{19}$. Somme toute, il aimerait bien qu'on le lise: il importe en effet, d'avoir un nom avant de rentrer au pays ${ }^{20}$. Il semble d'ailleurs qu'il ne faille pas s'illusionner sur le désintéressement de Blanchet dans le choix de cette carrière. Ne confie-t-il pas un jour à Neilson: «Je poursuis mes études non parce que j'y trouve du pláisir mâis pour éviter dé pilus grardis désagréments». ${ }^{21}$ Quoiqu'il en soit de son sentiment profond à l'égard de la médecine, c'est dans ce domaine qu'il choisit de faire carrière. De retour au Canada, il est d'ailleurs justifié de s'attendre à ce que sa présence soit remarquée. N'est-il pas depuis 1760 un des seuls Canadiens à être allé étudier à l'étranger? N'est-il pas aussi le premier médecin canadien à avoir écrit un traité médical qui fut vendu, bien que modestement en Amérique et en Europe ${ }^{22}$ ? Qui

\section{National Library of Medicine, Bethesda, Maryland.}

a) "On the vapour which rifes from the surface of the river St-Lawrence during the severe cold of winter. The New York Medical Repository, 3, no 2 (1800), art. 11: 154-155.

b) "Facts and remarks on the antiseptic powers of lexivial and oleaginous substances", Ibid., 2 (1800), no 2, art. 12: 156-160.

c) "Septic (nitric) acid the cause of the mortality among the neat cattle and horses of Canada, among their stabling in winter», Ibid., no 3 (1800), art. 7: 256-262.

d) «Theory and explosions», Ibid., 3, no 3 (1800), art. 8: 262-267.

19 Lettre à J. Neilson, 16 mars 1800 , ibid., 177. Il parle en fait ici de sa thèse. Cette cotisation ne rend pas bien compte des intentions de son travail. En réalité Blanchet veut développer un «modèle» original d'interprétation des lois de la nature qui se situerait au niveau des grands courants de pensée du XVIIIe siècle. (Cullen, Brown, E. Darwin). Blanchet cependant a fait peu d'observation; il fut davantage un philosophe qu'un homme de science.

20 Lettre à J. Neilson, 20 juillet 1800, ibid., 209.

21 Lettre à J. Neilson, 16 mars 1800, ibid., 177.

22 Lettre à J. Neilson, 20 juillet 1800, ibid., 210: «J'enverrai probablement la plus grande partie en Europe, vue que c'est là où l'on pourra apprécier le mérite ou le démérite de l'ouvrage. Comme le plan en est absolument neuf, j'ai hâte de voir quelle sensation il produira dans le monde scientifique.» 
d'autre que lui peut citer sa participation à une revue scientifique et à la plus célèbre association scientifique américaine et peut se glorifier d'avoir pénétré dans les milieux érudits de New York? François Blanchet s'est donc donné, durant ces années, une formation qui devait bientôt en faire une personnalité en vue de Québec, au moins dans le domaine médical.

\section{b) Les débuts professionnels}

Blanchet ne perd pas de temps. Dès son arrivée, on le voit préoccupé par le souci de se tailler une place au sein de l'élite médicale québécoise. Aussitôt après avoir obtenu la licence du Bureau des examinateurs ${ }^{23}$, il ouvre cabinet dans une maison qu'il a achetée, rue des Remparts, à l'intérieur des murs ${ }^{24}$. La manifestation de cette volonté d'ascension sociale se concrétise aussi par un brillant mariage, presque en même temps, avec Henriette Juchereau-Duchesnay, la fille d'un grand seigneur du Bas-Canada, l'honorable Antoine Juchereau-Duchesnay, écuyer, membre du Conseil privé et du Conseil exécutif et seigneur de Beauport. Celui-ci est suffisamment fortuné pour doter sa fille de la jolie somme de 20000 livres $^{25}$. Soucieux d'autre part de faire valoir ses talents professionnels, Blanchet publie, au cours des années 1801 et 1802, quelques articles scientifiques dans la Gazette de Québec que dirige John Neilson ${ }^{26}$. Sa thèse aussi a fait l'objet de publicité de même que son élection comme membre de l'American Philosophical Society de Philadelphie, nomination que lui ont value ses recherches sur la lumière ${ }^{27}$. C'est à cette époque aussi qu'il est nommé assistant-chirurgien de la milice, poste qui le plaçait dans une position de visibilité appréciable $^{28}$. Novateur, il met sur pied un enseignement et, en 1804, il com-

23 A.P.C. Mss, série S.RG 4 AL: «We have examined Mr. Francis Blanchet and we humbly conceive he may be licenced to practice Physic and Surgery».

24 Il s'agit d'une maison de pierre de taille à deux étages. A.N.Q.Q., Joseph Planté, 16 novembre 1802 , no 3189.

25 A.N.Q.Q. Joseph Planté, le 6 septembre 1802, no 3119. Le seigneur Duchesnay est aussi propriétaire d'un des plus importants moulins à farine de l'époque. Durant la seule année de 1788 , son moulin a converti près de 30000 minots de blé en farine. Sa seigneurie rapporte 1000 livres par an, ce qui constitue d'après Ouellet un des plus importants revenus seigneuriaux de l'époque. F. Ouellet, Le Bas-Canada, 1791-1840, changements structuraux et crise (Ottawa, Editions de l'Université d'Ottawa, 1976), 16, 18.

26 La Gazette de Québec, 24 décembre 1801: «cure of ulcers by a new practice»; 12 novembre 1801: «experiment as to the oxidation by pus», 6 mai 1802: «studies as to acidity in the atmosphere».

27 La Gazette de Québec, 5 mars 1801.

28 Il est attaché à l'état-major de la première division des milices de la ville et du district de Québec. Almanach de Québec (Québec, J. Neilson, 1806), 07-08. 
mence à donner chez lui des cours privés de chimie appliquée à la médecine aux aspirants à la licence en médecine ${ }^{29}$. Les choses vont donc bien pour Blanchet et ce, non seulement sur le plan professionnel, mais aussi en affaires. Grâce aux avoirs du couple et à ses revenus de médecin, le jeune Blanchet bénéficie, déjà à 29 ans, d'une aisance financière. Si bien qu'en 1805, il peut acheter sans difficulté une seconde maison dans la Haute-Ville, 19 rue des Pauvres, près de l'Hôtel-Dieu. Le couple s'y installera et louera celle de la rue des Remparts ${ }^{30}$.

Ce cheminement personnel, joint entre autres au contexte politique des années 1810-1820, devait bientôt conduire à une solide remise en question du sort de la médecine dans la colonie.

\section{II - RÉFORMER LA VIE MÉDICALE DE LA COLONIE}

On sait que la fin du XVIIIe siècle et le début du XIXe siècle marquent un moment important de l'histoire de la médecine et de ia profession médicale en Amérique du Nord. C'est l'époque où, aux Etats-Unis, on assiste à la fois à l'apparition des premières écoles de médecine (Philadelphie: 1765, Columbia: 1767, Harvard: 1783) et aux débuts de l'enseignement clinique dans les hôpitaux (New York: 1791, Boston: 1821). C'est également le moment de la création des premiers réseaux de communication tels les journaux, les bibliothèques et les sociétés médicales. C'est du reste de ces sociétés que naîtra aux Etats-Unis l'American Medical Association qui représente, aux yeux de J.H. Means, les débuts de la médecine organisée en Amérique ${ }^{31}$. Cette période marque aussi le commencement des débats relatifs à l'hygiène publique, lesquels trouveront leur concrétisation quelques décennies plus tard dans la création des Bureaux de santé. Influencé par ce mouvement, le Bas-Canada va connaître lui aussi, bien qu'un peu plus tardivement, un courant similaire qui vise sensiblement les mêmes objectifs. Blanchet, qui a pu juger de l'impact de la réforme aux Etats-Unis, sera bientôt un des initiateurs de cette réforme au Bas-Canada.

Au tournant du XIXe siècle, la seule loi qui régit la vie médicale reste celle de 1788. La composition du corps médical a considé-

29 La Gazette de Québec, 13 décembre 1804.

30 "A louer, une grande et belle maison à 2 étages, nouvellement réparée, sur la rue des Remparts». La Gazette de Québec, 7 février 1805.

31 J.H. Means, "Homo Medicus Americanus», in K.S. Lynn, The Professions in America (Boston, Beacon Press, 1969), 45-68 
rablement changé depuis la Conquête. Maintenant moins d'un tiers seulement des praticiens licenciés sont canadiens (c.a.d. francophones). La structure de cette profession est elle-même très différente. Ce sont maintenant les chirurgiens militaires britanniques qui tiennent le haut du pavé. Ce sont, dit-on, vers 1800 , les plus compétents, ils peuvent bénéficier d'une clientèle civile, ou pratiquer dans les hôpitaux et les institutions religieuses, et cela, sans avoir à se présenter devant le bureau des examinateurs de leur district. Ils forment du reste toujours la majorité des membres de ces bureaux. Ce sont eux aussi qui bénéficient des clientèles urbaines les plus fortunées ${ }^{32}$.

Le groupe des praticiens civils est composé d'une façon beaucoup plus hétérogène: on y trouve des Canadiens, des Britanniques, des Américains, des Allemands ${ }^{33}$. Ils ouvrent leur cabinet autant à la ville qu'à la campagne, bien qu'en général les Canadiens préfèrent s'établir en milieu rural. Ces praticiens civils n'ont d'ailleurs pas tous reçu une formation identique. Certains sont passés par une université; d'autres ont suivi quelques cours dans une école; mais la plupart ont reçu leur formation par apprentissage. D'ailleurs, après leur période de formation, ils ont tous reçu du Bureau des examinateurs de leur district, une habilitation spécifique les autorisant à pratiquer soit la chirurgie, soit la pharmacie, soit le métier d'accoucheur, soit deux des trois domaines, ou les trois. En fait, le titre de médecin diplômé fut réservé à quelques individus seulement parmi lesquels on trouve, chez les Canadiens, Fr. Blanchet, P. De Sales Laterrière et quelques autres.

En matière d'éducation, le Bas-Canada n'a toujours pas de collège d'enseignement de la médecine et, à part une infime minorité, rares sont ceux qui peuvent se permettre d'aller étudier à l'étranger. L'apprentissage reste donc en pratique le seul moyen de formation dans la colonie. L'enseignement clinique ne fait pas non plus partie du cursus de formation et les étudiants en médecine sont donc en général tributaires, pour leur formation, du savoir et de l'attention accordés par leurs maîtres. La situation hospitalière reste elle-même très archaïque. Le Bas-Canada ne compte toujours

32 Sur ces questions: B. Tunis, The Medical Profession in Lower Canada: its Evolution as a Social Group 1788-1838, B.A. with honours in history (Carleton Univ. May 1979), 164 p. «Pour quelles raisons, demande un lecteur du Canadien, les militaires ont-ils le droit de pratiquer sans licence? Pour quelles raisons ont-ils été jusqu'à présent à la tête de toute institution civile lucrative ou honoraire? " Le Canadien, 23 août 1817; voir aussi le 19 juillet 1817.

33 B. Tunis, ibid., 49. 
que cinq hôpitaux: un Hôtel-Dieu dans chacune des trois grandes villes de la province et un Hôpital Général à Québec et à Montréal. Ces hôpitaux ne comptent qu'une vingtaine de lits chacun. Des hôpitaux spécialisés, comme on commence à en voir aux EtatsUnis, n'existent pas et aucun n'est habilité à recevoir des malades contagieux. On ne trouve pas non plus de dispensaire, de sorte que les soins individuels à domicile ou chez le médecin constituent encore le type de soins le plus répandu. La situation qui prévalait au milieu du XVIIIe siècle à l'égard de l'hygiène n'a pas changé elle non plus. Il n'y a pas encore au Canada, comme cela commence à se voir, surtout en Europe, de politique d'hygiène sociale. Les seuls préceptes en ce domaine sont pratiquement laissés à l'initiative individuelle et familiale. Bien sûr, dans les villes, la police voit au bon ordre et à la propreté, mais son manque de connaissance en matière d'hygiène réduit considérablement son efficacité. D'ailleurs, les problèmes sont nombreux: la ville et surtout les quartiers populaires sont sales et les services, comme les égouts, inadéquats ${ }^{34}$. Une législation sanitaire efficace ne vient pas non plus exercer un contrôle sur les navires en provenance des Iles britanniques et qui sont souvent porteurs de maladies contagieuses. Les organismes d'assistance sociale sont toujours les mêmes et ils relèvent des communautés religieuses. Celles-ci, cependant, avec l'arrivée croissante dans les villes du nombre d'immigrants et de migrants venus de la campagne, suppléent de plus en plus difficilement aux besoins. L'État était intervenu lors des épidémies de la fin du XVIIIe siècle, mais cela n'avait pas donné lieu à la création d'organismes permanents responsables de la surveillance et de l'amélioration de l'hygiène publique (urbaine ou rurale) et de l'assistance aux personnes en difficulté. C'est donc cette situation que veut changer au Bas-Canada, un groupe de jeunes médecins du début du XIXe siècle au sein duquel figurent à Québec, les docteurs Fr. Blanchet, F.-X. Tessier, A.-G. Couillard, Jos. Painchaud, C.-N. Perrault, E.-P. Taché et des médecins civils anglophones comme les Dr W. Hall et J. Morrin de Québec. Ce groupe aura une action déterminante d'abord en ce qui concerne le développement de la profession elle-même, ensuite à l'égard des institutions d'enseignement, enfin en ce qui a trait à l'hygiène publique.

34 W. Kelly, «On the Medical Statistics of Lower Canada», Transactions of the Literary and Historical Society (1837): 210. 


\section{a) L'organisation de la profession médicale}

La profession médicale québécoise telle que nous la connaissons aujourd'hui trouve en grande partie ses racines au début du XIXe siècle. C'est elle qui, avec la profession légale, trace la voie aux autres professions qui se développeront par la suite.

La profession médicale bas-canadienne souffre, au tournant du siècle, de malaises sérieux: problème d'une profession «chapeautée» par des militaires, problème d'une clientèle difficile à gagner, problème aussi d'un groupe qui doit s'affirmer sur le plan du savoir, vaincre les préjugés qui pèsent sur lui et se donner des institutions d'enseignement. Aussi est-ce pour faire face à ces difficultés que des médecins commencent à se réunir et que sont fondés en 1818 la première société médicale et en 1826 le premier journal de médecine.

Fr. Blanchet qui a pris connaissance de l'existence de sociétés médicales aux États-Unis, où elles existent depuis les années 1760, est, avec F.-X. Tessier, le maître-d'oeuvre de cette première association au Québec.

À la suite de son expérience personnelle comme membre de la Société philosophique de Philadelphie, il croit aux bienfaits de ces sociétés pour le développement du savoir et l'unification de la profession. Enfin, Blanchet qui, d'autre part, oeuvre, depuis 1809, à la Chambre d'Assemblée ${ }^{35}$ pour des changements importants dans la profession, est profondément convaincu qu'il ne peut y avoir de progrès pour le groupe médical sans une amélioration de la formation de ses membres. L'association qu'un petit groupe de médecins et de chirurgiens décide de fonder en 1818 sous la présidence de Blanchet vise du reste essentiellement à «promouvoir les connaissances de la médecine en cette province ${ }^{36}$. L'histoire de cette pre-

35 Il sera député jusqu'en 1830, sauf pendant deux années.

${ }^{36}$ The Quebec Gazette, 20 mai 1818: «Society Formed at Quebec to Promote Medical Science», ibid., 28 mai 1818, 3: «Il y a eu une assemblée des médecins et chirurgiens de la ville à l'Hôtel de l'Union, lundi dernier donc: 25 mai. «Le docteur Blanchet à la Chaire». Après des débats sur la nécessité de former une Société, tout petite ou peu nombreuse futelle, pour promouvoir les connaissances de la médecine en cette province. Il a été résolu. Que les médecins et chirurgiens présents formeraient une Société de médecine, et qu'ils inviteraient leurs confrères de la campagne à correspondre avec eux, afin de connaître les cas extraordinaires dans leur profession; et il a été nommé un comité pour préparer et faire rapport à la prochaine assemblée, lundi le ler de juin, d'un projet de règles pour ladite société.» Frs Blanchet, M.D. 
mière société nous est mal connue mais il semble qu'elle cesse ses activités moins d'un an après sa création ${ }^{37}$. L'idée cependant devait être reprise avec plus de force et sur des bases plus solides en 1826. Le contexte dans lequel cette société revoit le jour est intimement lié à la création en 1826, par une quinzaine de médecins de Québec et Montréal, de la première revue médicale au Canada: Le Journal de Médecine de Québec ${ }^{38}$.

Alors que la société, elle, croit et vise au développement du savoir par une élite, le journal, lui, recherche une participation plus vaste. Il vise en particulier à créer des échanges entre les praticiens de la province. Outre une présentation des publications les plus importantes dans le secteur de la médecine en Europe et aux Etats-Unis, le journal consacre toujours plusieurs pages aux affaires locales. Une correspondance fait part, à chaque parution, des problèmes survenus dans la province dans le secteur médical. On y tient des statistiques sur la mortalité dans les hôpitaux ${ }^{39}$ et des réprésentiatiôñs sonint fáites au gouvernement concernant les mesures d'hygiène à prendre à l'égard des immigrants et les besoins de la population locale à l'égard des services de santé. Le journal n'eut pas cependant, pendant ses deux années d'existence, toute la participation désirée et, dans les faits, les collaborateurs furent presque toujours les mêmes.

Outre la recherche du développement des connaissances, la lutte pour le développement de la profession médicale s'inscrit dans le contexte d'une reconnaissance sociale du médecin civil. Un de leurs objectifs premiers est de faire en sorte que cessent de cohabiter au Bas-Canada deux médecines, l'une aux mains des Britanniques, notamment les militaires, et au service de l'armée, des élites

37 La Société est organisée suivant une structure précise que viennent confirmer des résolutions. Il est dit que la société vise à l'amélioration des diverses branches de la médecine: histoire naturelle, botanique, chimie, pharmacie, physique, chirurgie, anatomie, physiologie, jurisprudence médicale, police médicale, obstétrique. La société se réunit tous les lundis du mois et tous les membres doivent y assister. De même, tous doivent présenter une communication à tour de rôle. Journal de médecine de Québec, 1 janv. 1827, 106. Voir aussi «Histoire de la Société médicale de Québec», Laval médical, 8, no 1 (janv. 1943): $63-121$.

38 Parmi les membres fondateurs on remarque, outre le directeur F.-X. Tessier, à Québec: Jos Painchaud, Jos. Parent, Jos. Morrin, Fr. Blanchet, C.-N. Perreault, P.-M. Bardy et W.A. Hall; à Montréal; Wm. Caldwell, W. Robertson et J. Stephenson; les Dr Meilleur de l'Assomption et W. Talbot de Trois-Rivières en font aussi partie. J.J. Heagerthy, Four Centuries of Medical History (Toronto, MacMillan, 1928), 276.

$39 \mathrm{Fr}$. Blanchet, entre autres, y présente en avril 1827 un article sur les admissions à l'hôpital des Emigrants du ler mai au ler août 1826. Journal de médecine de Québec (avril 1827): $210-213$. 
et des institutions; l'autre, civile, au service de la masse. Ils veulent briser cette frontière et instituer une profession unique où la promotion serait le fruit des qualités individuelles et non plus du patronage. Ce mouvement critique s'inscrit d'ailleurs dans le climat général inspiré par le Parti canadien qui anime alors le Québec. "S'il fut un temps, rapporte un lecteur du Canadien, où il était indispensable de se servir de ces messieurs, ce temps-là est fini, nous avons maintenant des praticiens civils capables de faire honneur aux situations qu'ils occupent. $\gg^{40}$ Le savoir même des militaires devient contesté. En fait plusieurs d'entre eux n'avaient pas passé les examens du Collège royal des chirurgiens, mais avaient obtenu leur titre et leur droit à la pratique à la suite de trois années de service dans l'armée ou la marine royale ${ }^{41}$.

Un autre point de revendication vise à changer la composition du Bureau des examinateurs dont les membres sont nommés par le gouverneur. Ce Bureau en effet centralise des pouvoirs importants: c'est lui qui étudie les candidatures et soumet ses choix au gouverneur qui entérine ordinairement leur décision. Or ce Bureau, comme plusieurs autres organismes coloniaux, n'est absolument pas représentatif. Non seulement les francophones y sont tenus à l'écart ${ }^{42}$, mais on se plaint aussi que ce Bureau, créé lors de la loi de 1788, a favorisé surtout les chirurgiens militaires (qui sont reçus sans examen) au détriment des médecins et chirurgiens civils et surtout francophones ${ }^{43}$. On se plaint de plus en plus de leur intransigeance. Peu auparavant, par exemple, deux candidats, R.-S. Bourdages et T. Fortier, bien qu'ils aient été dûment diplômés de l'Uni-

40 Le Canadien, 2 août 1817. Texte signé, signale l'auteur de l'article, par tous les praticiens civils de la ville de Québec, sauf deux.

41 M.J. Readers rapporte en effet dans une étude récente que les qualifications requises pour devenir chirurgien dans l'armée ou la marine étaient inférieures à celles nécessaires pour obtenir le diplôme ordinaire du Collège royal des chirurgiens, mais après trois années de service dans l'armée ou dans la marine un chirurgien pouvait s'orienter dans la pratique civile au même titre que les autres membres du Collège. Professional Men, the Rise of the Professional Classes in the 19th Century (N.Y. Basic Book, 1972), 35. D'autre part, d'après Carr-Saunders, les standards requis au début du XIXe siècle pour pratiquer la chirurgie en Angleterre étaient bien en deçà de ceux exigés pour devenir médecin; à son avis, ils étaient même «deplorably low». The Professions (London, Frank Cass and Co., 1964), 82. Enfin, d'après Mrs Jeanne Peterson, "the services had the reputation for not at racting the ablest and best students». The Medical Profession in Mid-Victorian London (Berkeley, University of California Press, 1978), 125.

42 A. Jobin, "Histoire de la médecine dans le Bas-Canada», Bulletin de la Société médicale des hôpitaux universitaires de Québec (1932), \#9: 38; B. Tunis op. cit., 57.

43 Pétition de médecins de Québec adressée au Gouverneur, Journal de la Chambre d'Assemblée du Bas-Canada, 27, 21 janv. 1818, 44. Voir aussi B. Tunis, op. cit., 79. 
versité de New York, se sont vus demander de passer un examen devant le Bureau parce qu'ils n'avaient pas reçu leur diplôme d'une université britannique ${ }^{44}$. On revendique une nouvelle composition de ce Bureau. Des modèles sont proposés, tel celui soumis en 1823 à l'Assemblée et qui sera entériné presque textuellement en 1831. Un groupe de médecins du district de Montréal a soumis à la Chambre par le biais d'un comité dont fait partie Blanchet le projet que ce bureau soit dorénavant composé de 20 ou 30 membres choisis par une assemblée générale de médecins et chirurgiens et que ce soit lui qui choisisse parmi ses membres neuf examinateurs, élus pour un certain nombre d'années et qui feraient passer en public les examens des candidats à la licence ${ }^{45}$.

Cet esprit de revendication et de solidarité des médecins civils face aux médecins militaires se manifestera aussi en 1817 lors de la création du bureau de vaccination. Un bill voté à la Chambre stipulait que les six médecins que choisirait le gouverneur devraient être des civils. Celui-ci nomma finalement quatre médecins anglonhones, un militaire et un seul francophone, François Blanchet, comme vice-président. Ce dernier déclina l'offre et demanda, étant un des plus vieux médecins de la ville, à être nommé président, ce qui lui fut refusé. Il donna alors sa démission et, par esprit de solidarité, aucun médecin francophone n'accepta de siéger au comitét6.

Outre la contestation du pouvoir colonial, les médecins civils cherchent aussi à étendre leur influence sur un autre secteur: celui de la médecine en milieu populaire. Ce secteur, du reste, ils ne l'ont jamais vraiment occupé mais il leur échappe largement à la fin du XVIIIe siècle, suite à l'absence généralisée de médecins qualifiés dans les campagnes. A leur avis, les populations rurales sont entre les mains d'autodidactes qui agissent en véritables meurtriers. Ce problème du contrôle de l'exercice de la profession n'était pas nouveau et c'est pour obvier à ce malaise qu'avait été promulguée la loi de 1788. Cette loi est, à leur avis, inopérante et «elle n'a pas empêché les charlatans de proliférer dans les campagnes, ce qui empêche les jeunes gens bien instruits de s'y établir ${ }^{47}$. De nombreuses pres-

44 Le Canadien, 20 juin 1818. Ils s'en plaindront au Conseil exécutif et obtiendront finalement gain de cause. Bourdages avait fait son apprentissage chez Blanchet. Seuls les diplômés des universités britanniques n'avaient pas à passer cet examen devant le Bureau des examinateurs.

45 Journaux de la Chambre d'Assemblée du Bas-Canada, 9 décembre 1823, 40.

46 Le Canadien, 19 juillet 1817.

47 Lettre d'un lecteur, Le Canadien, 23 août 1817. 
sions avaient été exercées depuis le début du siècle pour reformuler la loi sur cette question mais ce n'est qu'en 1814 que la Chambre d'Assemblée accepta, après les revendications de Blanchet, Bourdages et Borgia, de former un comité pour préparer un bill qui amenderait l'ordonnance de $1788^{48}$ et autoriserait un contrôle plus strict sur les praticiens illégaux. Afin de mettre sur pied une surveillance plus efficace, on demande alors à la Chambre d'accorder des pouvoirs plus grands aux juges de paix.

Parallèlement à cette lutte contre le «charlatanisme», le corps médical s'inscrit aussi, à certains égards, en conflit avec le groupe des pharmaciens. Afin de contrecarrer l'action des pharmaciens avec lesquels le corps médical est en lutte pour la clientèle dans les campagnes et dans les quartiers populaires, il est notamment demandé à la Chambre d'interdire aux pharmaciens de visiter les malades et de prescrire des médicaments «comme ils ont l'habitude de le faire ${ }^{49}$ car cela le prive d'une clientèle qu'il juge de sa juridiction. Les médecins requièrent aussi pour l'organisme de contrôle qu'ils veulent mettre sur pied le pouvoir de visiter les pharmacies et les laboratoires des apothicaires et des chimistes au moins une fois l'an afin de s'assurer qu'ils ne vendent pas de médicaments falsifiés ou trop vieux.

Enfin, autre point de revendication, ils réclament des tarifs uniformes d'honoraires ${ }^{50}$ afin d'éviter une trop vive concurrence à l'intérieur de la profession et assurer à tous une stabilité de revenus.

\section{b) La quête d'institutions de formation}

Le développement d'un système d'éducation devait également bientôt faire partie des réclamations de ce groupe de médecins. Maintenant que le corps médical fait montre de son intention de développer le savoir, l'étape suivante, logiquement, devrait être l'établissement d'institutions d'enseignement et de recherche. Pour tous, en effet, il était devenu évident que le niveau de la formation professionnelle ne pourrait être accru par la seule voie de l'apprentissage, car ce système ne fournissait pas assez en ce qui concerne l'étude des principes théoriques et de la méthode scientifique.

48 Journaux de la Chambre d'Assemblée du Bas-Canada, 28 janvier 1814 et ler février 1814, 123-137-139; pétition présentée par Blanchet.

49 Journaux de la Chambre d'Assemblée du Bas-Canada, 9 déc. 1823.

50 C.-A. Gauthier, op., cit., 97. 
Il n'y a pas avant les années 1820 d'école de médecine au BasCanada, et les hôpitaux sont très peu utilisés pour la formation clinique des étudiants. L'apprentissage reste donc pour ceux qui ne peuvent pas se permettre de poursuivre des études à l'étranger, le seul moyen de formation. Une première tentative pour créer une école de médecine avait eu lieu à Québec en 1798; mais elle s'était soldée par un échec par suite, pense-t-on, entre autres raisons, des réticences des autorités face à la dissection et à l'étude de l'anatomie ${ }^{51}$. Convaincus de la nécessité d'instituer des cours pour les étudiants, certains médecins commencent à donner des cours privés. Un des premiers en ce domaine est François Blanchet qui donne, à partir de 1804 , des cours privés de chimi $^{52}$. Des cours semblables semblent également offerts à Montréal à la fin des années 1810. Le besoin d'intégrer ces cours à l'intérieur d'un cadre institutionnel se faisant sentir avec de plus en plus d'insistance autour des années 1815-1820, deux voies paraissent possibles: celle des dispensaires et celle des hôpitaux. Il semble que ce soit à Québec et par i'intermédiaire d'un groupe de médecins francophones que les premiers cours dans les dispensaires sont créés dans les années 1810 et $1820^{53}$. En 1823, François Blanchet et Anthony von Iffland créent le Dispensaire de Québec afin d'y enseigner l'anatomie, la chimie, la médecine et la chirurgie ${ }^{54}$.

Il existait une autre possibilité, celle d'intégrer ces cours dans les hôpitaux. Déjà, en 1816, Blanchet et Bourdages avaient piloté, sans succès, un projet de loi à cet effet à la Chambre ${ }^{55}$. Mais on garde confiance et lorsque, peu après, on projette la création de l'hôpital des Emigrés, plusieurs sollicitent auprès du gouvernement l'autorisation de pouvoir mettre à la disposition des étudiants des cours d'anatomie, de chirurgie, de pharmacie et «sur l'art d'accoucher $^{56}$. Le projet sera finalement accepté en 1825 alors que la loi

51 C.-A. Gauthier, op cit., 101.

52 Gazette de Québec, 13 décembre 1804 et 20 novembre 1806: «Le cours portera non seulement sur les gaz et sur les ingrédients qui composent les matières animales et végétales, mais encore on y traitera des productions naturelles du pays telles que des montagnes, des mines, des putréfactions, des lacs, des rivières et de la formation des eaux minérales, etc.»; voir aussi Le Canadien, 9 janvier 1808.

53 Annonces dans le journal Le Canadien à partir de 1818. Les cours ont lieu le soir et sont dispensés entre 1818 et 1819 par les Dr Perrault, De Sales Laterrière, Mercier et Von Iffland.

54 C.-A. Gauthier, op. cit., 101.

55 B. Tunis, op. cit., 99.

56 Le Canadien, 2,16 et 30 déc. 1818. 
votée prévoit que les médecins attachés à cet hôpital (Morrin, Blanchet, Perrault, Painchaud, Hall) pourront y donner un enseignement $^{57}$.

Les médecins anglophones de Montréal revendiquent aussi dans le même sens et lorsqu'on prévoit la construction du Montreal General Hospital, il ne fait pas de doute à l'esprit des fondateurs que cette institution doit servir à la formation des étudiants. Aussi, peu de temps après l'ouverture de l'hôpital, une résolution est-elle adoptée par laquelle les Dr Stephenson, Holmes, Caldwell, Robertson et Loedel sont autorisés à commencer un enseignement dans cette institution ${ }^{58}$. C'est d'ailleurs de cette école, The Montreal Medical Institution, que devait voir le jour le collège de médecine de McGill en 1829. Bien que moins fructueuses, les requêtes pour la création d'une institution d'enseignement semblable se font également pressantes chez les francophones dans les années 1820. A Québec, par exemple, les étudiants en médecine, qui se sont récemment regroupés en association, soumettent eux-mêmes une demande en ce sens au gouverneur. Ils demandent une aide de l'administration "pour acheter et construire une maison où seraient données des leçons publiques sur la chimie, la médecine et d'autres branches de la philosophie, afin qu'ils puissent avoir toutes les facilités sans être dans la nécessité d'aller dans d'autres pays pour acquérir des connaissances qu'ils peuvent aussi bien acquérir dans le leur» ${ }^{59}$. La fondation d'une telle institution à Québec devait cependant être beaucoup plus lente puisque finalement la première école de médecine ne sera créée dans cette ville qu'en $1845^{60}$.

57 Journaux de la Chambre d'Assemblée du Bas-Canada, 34 (1825), app. I; voir aussi ibid., 15 fév. 1823 .

58 «The medical officers of the Montreal General Hospital having seen the great difficulties which the student of medicine in Canada has to encounter before he can acquire a competent knowledge of his profession; knowing the inconvenients resulting to many from the necessity at present existing of spending several years in a foreign country to complete a regular medical education and considering that the present establishment of the Montreal General Hospital affords the student a facility of acquiring a practical knowledge of physic never enjoyed in these provinces, and that his advantage will be greatly enhanced by delivering courses of lectures on different branches of the profession, held a meeting to consider of the practicality of founding a medical school in this city". H.E. MacDermot, A History of the Montreal General Hospital (Montreal, 1950), 19.

59 Journaux de la Chambre d'Assemblée du Bas-Canada, 3 février 1826.

60 L'inauguration officielle de l'Ecole de médecine de Québec n'eut lieu que le 15 mai 1848. Chs.-M. Boissonnault, Histoire de la faculté de médecine de Laval (Québec, P.U.L., 1953), 137. 


\section{c) L'hygiène publique: des services à créer}

Le début du XIXe siècle marque également une étape importante de l'histoire de l'hygiène publique au Québec. La prise en charge de ce secteur cesse de plus en plus d'être assurée, au gré des besoins, par des associations de volontaires, par les communautés religieuses ou par le clergé, pour passer entre les mains de l'État et du corps médical. A la suite des sérieuses épidémies de petite vérole qui sévissent à la fin du XVIIIe siècle, l'Etat est appelé à jouer un rôle accru. Ce rôle sera encore accentué, au début du XIXe siècle, en raison des problèmes soulevés dans les villes de Québec et Montréal par l'arrivée croissante des ruraux et surtout par les immigrants britanniques qui débarquent de plus en plus nombreux. Cette vague migratoire, que le Bas-Canada n'a du reste pas le choix de repousser, est majoritairement constituée d'une population démunie et souvent malade. Le niveau de conscience à l'égard de l'hygiène publique est aussi favorisé à partir de 1791, par la publication dans les Iournoux de la Chambre d'Assemblée, d'un nouveau type d'iñformations au Canada: les statistiques vitales. Des comparaisons sont maintenant possibles entre les taux de mortalité du Bas-Canada, du Haut-Canada, de la Grande-Bretagne et d'autres pays. C'est de cette période que datent des mesures comme la vaccination, la création du premier conseil d'hygiène, l'institution des mesures plus strictes pour l'observation de la quarantaine et l'apparition des dispensaires et des premiers hôpitaux spécialisés.

Durant ces années marquées par les épidémies, la prévention des maladies devient une préoccupation essentielle dans la colonie. C'est vers 1802 que la vaccination fut introduite au pays par les autorités du Bas-Canada. Au cours des années suivantes, elle connaît un encouragement presque inconditionnel de la part des médecins et même des membres du parlement, et des subsides importants sont votés pour sa diffusion dans la population. Un comité sera mis sur pied à cet effet en 1817. Â plusieurs reprises, depuis les années 1810 , des projets sont présentés à la Chambre par Lee, Blanchet et quelques autres députés. Devant ces pressions, le gouverneur se voit forcé, en 1817, de nommer un comité spécialement chargé d'encourager «l'inoculation de la vaccine» et François Blanchet est désigné à ce comité avec 5 autres médecins importants de Québec, T. Fargues, W. Harkett, J. Cockburn, J. Morrin et D. Clarke. Il s'en désiste en juillet 1817 pour les raisons évoquées plus haut. Un vaste projet est alors mis en oeuvre pour la vaccination de toute la population bas-canadienne. Des fonds importants sont débloqués et une 
importante campagne d'information est lancée, entre autres par l'intermédiaire des curés, pour encourager la population à se faire vacciner contre la petite vérole ${ }^{61}$.

La deuxième mesure prise pour la prévention des infections fut le rétablissement des quarantaines. La création de la première station de quarantaine semble dater de 1721 à l'Ile-aux-Coudres ${ }^{62}$. La loi fut renouvelée en 1759 par le gouverneur suite à une nouvelle épidémie de petite vérole, mais elle devait être très peu appliquée dans la période qui suivit. En 1795, c'est la Chambre d'assemblée qui adopta elle-même, pour la première fois, un acte pour obliger les navires «venant des places infectées de peste ou d'aucune maladie pestentielle de faire quarantaine, pour empêcher la communication d'icelles au Bas-Canada ${ }^{63}$. La loi fut renouvelée en 1800 et en 1802 et même si en 1817 le gouverneur accorda plus de pouvoir aux officiers afin de mieux faire observer les règlements de quarantaine, elle resta malheureusement, toujours faute de moyens, relativement inopérante et inefficace.

C'est de cette époque également que datent les premières institutions destinées à enfermer sous un même toit les malades contagieux. Devant le refus des Hôtel-Dieu et des Hôpitaux Généraux à admettre ce type de malades et vu la gravité de la situation dans les années 1810, il fut décidé de créer une institution qui leur serait réservée: l'hôpital des Emigrés de Québec. Blanchet fut un des leaders de ce projet et c'est d'ailleurs sur sa proposition que la création de cet hôpital fut autorisée par la Chambre d'Assemblée en $1816^{64}$. On le retrouvera d'ailleurs directeur de cet hôpital en $1830^{65}$. Bien que les problèmes soulevés par l'arrivée des immigrants fussent moins aigus à Montréal, le besoin de ce genre d'institution spécialisée s'y faisait également sentir. C'est, entre autres, à cette fin que fut créé, en 1819, le Montreal General Hospital où des salles spécialement réservées à ce genre de malades furent conçues. C'est aussi dans cette optique qu'apparaissent les premiers dispensaires de Québec et de Montréal. Ces établissements de bienfaisance, dont nous ne connaissons pas bien le nombre, servent de lieux de consul-

61 Un long débat suivra en 1819 sur les activités et les dépenses de ce comité dans les Journaux de la Chambre.

62 Annuaire du Québec (Québec, Bureau de la statistique du Québec, 1967), 352.

63 Statuts provinciaux du Bas-Canada (1795), chap. V: 142-150.

64 Journaux de la Chambre d'Assemblée du Bas-Canada, 27 janvier 1816. Le premier hôpital des Emigrés fut élevé en 1817 à l'Ile-aux-Reaux, il sera peu après aménagé à la Pointe-I évis puis dans le faubourg Saint-Jean.

65 A.P.C., R G 4 s I, volume 326. 
tation ainsi que de lieux de distribution des médicaments aux malades pauvres. C'est là aussi que les apprentis médecins font leurs premières armes.

Une autre création de l'époque en matière de santé est celle du comité sanitaire de 1823 qui préfigure, d'une certaine manière, les futurs bureaux d'hygiène. Ce comité dont l'existence dura de mars à septembre 1823 et dont Blanchet fait partie fut chargé de mettre sur pied les moyens de lutte contre les épidémies dans la ville de Québec et d'assurer l'assistance médicale aux immigrants ${ }^{66}$. Des bureaux semblables seront créés lors de la dure épidémie de choléra en 1832; ils deviendront permanents à Québec en 1840 et à Montréal en 1851 .

$$
* * *
$$

Telle fut en gros l'action menée par ce groupe dont fait partie François Blanchet, dans le secteur médical. Ce groupe est constitué, en grande partie, de médecins civils qui, se considérant lésés, revendiquent leur place dans la société. Sûr de ses compétences et fort du sentiment d'appartenance que lui confère son association en formation, ce groupe critique le monopole constitué par les médecins militaires britanniques et cherche à évincer ces derniers des postesclefs. Parallèlement à ces objectifs, il demande la création d'institutions d'enseignement pour le développement et la standardisation du savoir médical. Il vise aussi au contrôle interne et externe de la profession par l'élaboration d'un code d'éthique, un contrôle plus strict des aspirants au titre de licencié, et un droit d'exercice exclusif aux seuls médecins reconnus. Enfin, son but primordial est de persuader la Chambre d'Assemblée et le gouverneur de voter une loi qui lui conférerait un plus grand pouvoir sur la profession, objectif qui sera partiellement réalisé en 1831 et, d'une façon encore plus claire en 1847, lors de la création du Collège des médecins et chirurgiens de la province de Québec. Ces revendications derrière lesquelles se dissimule un désir tant de reconnaissance sociale que d'amélioration financière, il les légitime en invoquant son souci de mieux servir la population. Ce souci, il l'exprime entre autres par le biais de son engagement dans les problèmes relatifs à l'hygiène publique. En revendiquant le développement de ce secteur, il cultive aussi l'espoir que s'ouvre à lui un nouveau champ d'action, par lequel pourra se renforcer le caractère utile d'une profession au service de l'humanité. Enfin, par le biais de ce groupe formé en bonne partie

66 Y siègent également les Dr Holmes, Laterrière, Morrin, Hall, Parant et Lloyd. 
de francophones, nous voyons que la participation croissante des médecins francophones à la vie médicale ainsi que leurs revendications s'inscrivent dans le mouvement général d'émancipation et de promotion de la bourgeoisie professionnelle canadienne-française et même de la société francophone dans sa globalité.

Comment finalement évaluer le rôle de François Blanchet dans cette réforme? Bien sûr, Blanchet n'a pas été le premier ni le seul à lancer un mouvement de cette importance. Des courants semblables, nous l'avons vu, existent depuis longtemps. Qu'on se souvienne de John Morgan et de son projet, dès 1765, pour rehausser le niveau et le statut de la profession médicale américaine. De même, au Canada, des groupes et des individus revendiquent depuis longtemps une législation plus favorable pour les praticiens légalement reconnus. L'acte de 1788 s'inscrit d'ailleurs dans cette démarche, mais ce n'est qu'au début du XIXe siècle qu'elle connaîtra son vrai démarrage.

Si Blanchet, comme on l'a vu, a été un élément catalyseur au sein de ce groupe, c'est qu'un ensemble de circonstances lui a permis de jouer ce rôle. En plus d'être très motivé par ce projet, Blanchet est un des seuls praticiens à être porteur de titre de médecin et à avoir écrit sur la médecine, ce qui confère une certaine notoriété et un respect dont ne jouissent pas la plupart de ses confrères. Il y a, en effet, très peu de médecins diplômés dans la colonie ${ }^{67}$ même en milieu anglophone ou chez les militaires. De plus, Blanchet est un homme informé. Il a voyagé et il a vu aux Etats-Unis les efforts faits, dans les grandes villes comme Boston, Philadelphie et New York, pour essayer de donner à cette profession un statut meilleur. Blanchet a également une bonne connaissance des milieux médicaux canadiens; chez Fisher il avait l'habitude de rencontrer des personnalités du milieu, autant militaires que civiles, de sorte que leur monde ne lui est pas étranger. Enfin, grâce à son poste de député à la Chambre où il est du reste, jusqu'en 1824, un des seuls représentants du monde médical et un des plus écoutés, il est souvent appelé à piloter des projets de loi concernant la médecine, et à y être en quelque sorte le porte-parole de ce groupe ${ }^{68}$. A la lecture de ce texte, le lecteur a peut-être l'impression que Blanchet fut avant tout quelqu'un qui trouva dans ce projet le moyen de promouvoir

67 Presque tous sont chirurgiens.

68 Nombre de médecins à la Chambre; 1809 à 1814: 2, 1815 à 1824: 5, 1825 à 1830: 10. B. Tunis, op cit., 93 . 
essentiellement ses propres intérêts, fussent-ils même simplement financiers. Ce serait trop simplifier ses motivations.

Blanchet, on le sait, possède des biens et il vit à l'aise; il est par ailleurs, un des seuls médecins diplômés de la ville et il aurait pu vivre assez facilement de sa seule pratique professionnelle; enfin c'est un homme connu, il est député et il tire d'autres revenus de ce poste. A mes yeux, c'est plutôt parce qu'il est en position de force et parce que sa carrière et sa situation économique sont assurées que Blanchet peut se permettre de poser des actes susceptibles de générer des conflits alors qu'ils ne lui rapporteront en fait pas tellement personnellement. Ses actions s'inscrivent donc dans un cadre plus vaste qu'au simple niveau individuel: c'est-à-dire au niveau professionnel, politique et social.

$\mathrm{Au}$ niveau professionnel, nous l'avons vu, ses actions sont solidaires du groupe auquel il appartient. Au niveau politique elles visent à briser les monopoles constitués par les Britanniques. On sait que Blanchet a été très actif en politique et qu'il s'est opposé à l'administration britannique à maintes reprises. On se souvient, entre autres, qu'il a été l'un des fondateurs du journal le Canadien et qu'il fut emprisonné pour «trahison» lors de la crise de $1810^{69}$. Sa participation à cette réforme s'inscrit dans la continuation de cette démarche. Blanchet est enfin un réformateur à la manière des «hommes éclairés» du XVIIIe siècle. Il veut un monde meilleur. Il cherche, entre autres, à moderniser la médecine du Bas-Canada, c'est-à-dire à la mettre au pas des grands pays pour en faire profiter «l'humanité», selon l'expression de l'époque. C'est aussi dans cette perspective, je pense, qu'il faudra situer sa participation aux débats sur le développement de l'éducation, des communications et de l'aide aux habitants des campagnes ${ }^{70}$.

69 Voir à ce sujet: J.P. Wallot, Un Québec qui bougeait, Trame socio-historique au Tournant du XIXe siècle (Québec, Boréal Express, 1973), chap. IV. du Canada.

${ }_{70}$ Une biographie de François Blanchet paraîtra dans le Dictionnaire biographique 